\title{
Implementing Coastal Inundation Data with an Integrated Wind Wave Model and Hydrological Watershed Simulations
}

\author{
Dong-Sin Shih ${ }^{1, *}$, Tai-Wen Hsu ${ }^{2}$, Kuo-Chyang Chang ${ }^{3}$, and Hsiang-Lan Juan ${ }^{3}$ \\ ${ }^{I}$ Taiwan Typhoon and Flood Research Institute, National Applied Research Laboratories, Taichung, Taiwan \\ ${ }^{2}$ Department of Hydraulic and Ocean Engineering, National Cheng Kung University, Tainan, Taiwan \\ ${ }^{3}$ Water Resources Agency, Ministry of Economic Affairs, Taipei, Taiwan
}

Received 29 September 2011, accepted 3 May 2012

\begin{abstract}
Coastal inundation due to wave overtopping coastal structures and storm surges often causes serious damage and danger to the population of Taiwan. Ascertaining the areas that are prone to coastal inundation is essential to provide countermeasures for mitigating the problem. Simulations without precipitation are examined in this study since overtopping has been determined to be a controlling factor in coastal flooding. We present scenarios for the simulation of coastal flooding with a unified wind wave and hydrological watershed model. The eastern coastal areas in Taiwan are selected as the study area. Simulations show that the resulting waves and tidal levels, generated by the Rankin-Vortex model and wind wave calculations, can be successfully obtained from the input data during wave overtopping simulations. A watershed model, WASH123D, was then employed for surface routing. The simulations indicate that the low-lying Yilan River and Dezikou Stream drainage systems were among the primary areas subject to inundation. Extensive inundation along both sides of the river banks was obtained in the case of extreme overtopping events. Coastal inundation could occur between the estuary of the Meilun Stream and most of the Nan-Bai-Bin areas in Hualien City. Calculations showed that more croplands flooded than did residential regions. No further damage to the upland of Hualien City was identified. The remaining study sites, Sinchen Township, Taitung City and Taimali Township, would only be slightly affected by seawater run-ups. Inundations caused by overtopping under extreme circumstances only affected the coast with almost trifling influence inland.
\end{abstract}

Key words: Wind wave model, Hydrological watershed model, Coastal inundations

Citation: Shih, D. S., T. W. Hsu, K. C. Chang, and H. L. Juan, 2012: Implementing coastal inundation data with an integrated wind wave model and hydrological watershed simulations. Terr. Atmos. Ocean. Sci., 23, 513-525, doi: 10.3319/TAO.2012.05.03.01(WMH)

\section{INTRODUCTION}

Typhoon related damage is among the most crucial disaster triggered issues confronted by the people of Taiwan. An average of 3 to 4 typhoons hit Taiwan from July through October (a typical typhoon season in Taiwan) every year producing massive amounts of precipitation over the island. Not only the dangers of flooding due to heavy precipitation, but also the likelihood of coastal inundation due to the overtopping of costal structures needs to be clarified. Typhoon induced coastal inundation could bring disaster to the people of the island. According to the official government website (http://www.wra.gov.tw/), Taiwan is surrounded by ocean with almost 1200 kilometers of coastline. Increasing

\footnotetext{
* Corresponding author

E-mail:dsshih@ttfri.narl.org.tw
}

sea level may be aggravated due to a global rise in the sealevel rise associated with climate change, tidal variation, and storm surges. Wave overtopping attacks the regions along the coast with seawater intrusion affecting low-lying villages especially during typhoon seasons. The occurrence of surface flooding obstructed by increasing sea-levels is also frequent. In short, storm surge and overtopping often cause serious damage and endanger the population of the island. Securing coastal areas from flooding has become an important task in Taiwan. Ascertaining the areas that are prone to coastal inundation will help in planning countermeasures for mitigation of coastal flooding. In order to investigate coastal inundation due to overtopping, precipitation is not taken into account in the simulations since overtopping has been determined to be the controlling factor. The main focus 
in this paper is simulating scenarios of extreme occurrences of coastal inundation e.g., intrusion of seawater into coastal areas or storm surge effects. We investigate several sites along the eastern coastal areas of Taiwan.

\section{METHODOLOGY}

Simulations are conducted of coastal inundation based on a unified wind wave and hydrological watershed model. The wind wave model is used to generate different return periods for storm surge as boundary forcing. Overtopping of coastal structures such as vertically walled jetties by solitary waves was applied because the periodic wave modeling was invalid (Synolakis 1987). Simulation domains were discretized with the finite element method for watershed modeling. An integrated multimedia, multi-process, physics-based distributed watershed model, WASH123D, with various spatial-temporal scale computations was implemented. The methodology is described in greater detail below.

\subsection{Wind Wave Estimations}

Coastal wave run-ups are among the most important factors considered in the design of jetties. A good index for evaluating the possibility of overtopping and overflow is needed. Empirical formulas have been used most often to determine the flow rates of wave run-ups, although on occasion, numerical approaches are applied for model simulation. The overtopping stress on each embankment should be less than $0.05 \mathrm{~cm}$ per meter to be considered safe. Overtopping and wave run-ups are closely related. This paper presents a wind wave model for the purpose of determining the amount of wave run-up and overtopping along the coast. The results of analyses of wave run-ups and scenarios of overtopping are provided for boundary forcing for watershed modeling. We evaluate the protection of embankments along the eastern coast under attack given various return periods during extreme circumstances. Generally speaking, the coastal regions in Taiwan are vulnerable to storm surges, overtopping, and seawater intrusion. Simulations of extreme situations are studied in this paper. In addition, practical countermeasures for mitigation of coastal inundation are proposed.

A numerical model is adopted in which the Reynolds averaged Navier-Stokes equation (RANS) is solved (Hsu et al. 2009). A low-Reynolds number turbulent model ( $\mathrm{k}-\varepsilon$ model) is further applied to capture the characteristics of water flow (Hsu et al. 2011). The immersed boundary method is used to simulate the complex events that occur as waves approach the coast. We aim at modeling the circumstances that occur as the seawater interacts with the bottom topography and coastal protection. The particle level set method (PLSM) is also used to capture the variations in wave shape near the coast (Enright et al. 2002). The inclusion of data assimilation techniques into the wind wave model makes the sequential simulation more applicable (Hsu et al. 2011). This is a necessary process for Taiwan due to its rapid change in coastal terrain. Run-ups, overtopping and breaking of waves are simulated in our modeling. In order to precisely evaluate the amounts of the run-ups, the initial basic water levels due to astronomical tides and storm surges with various return periods are assumed. Statistics are utilized to determine adequate boundary conditions. The wave heights and corresponding return periods are calculated. We then model the situation at embankments along the coast in extreme case scenarios.

We establish a model designed to simulate various scenarios of wave run-ups and overtopping in eastern Taiwan. After we decide on the study sites, digitized terrain information and aerial photographs are incorporated to determine the representative coastal embankments. Simulations using the collected information to calculate maximum run-ups are conducted. Then the possibilities of overtopping in eastern Taiwan are consequently obtained.

\subsection{Hydrological Watershed Calculations}

We conduct simulations of coastal inundations using the WASH123D numerical model for surface routings. The WASH123D numerical model was developed to include the flows of dendritic river/stream/canal networks, overland regimes and subsurface media. It is also an integrated multimedia, multi-process, physics-based computational model for watershed scale hydrology. We selected the WASH123D numerical model as the most appropriate protocol to conduct our research because it is designed to simulate the flow and transport processes in simulation problems with various spatial and temporal scales as long as the continuum assumptions are valid. Moreover, it is also capable of implementing the unsteady flows that invade a watershed (Yeh et al. 2006).

The theoretical fluid flows and transport processes built into the WASH123D numerical model are based on the conservation laws of fluid, momentum, energy and mass. They are associated with the constitutive relationships between the fluxes and state variables and appropriately formulated equations for source/sink terms. For modeling fluid flows in river/canal/stream networks, the one-dimensional Saint Venant equations can be modified to include the effects on density due to temperature and salinity. These are, in fact, the cross-sectional areas averaged from Navier-Stokes equations. Surface runoff over the region is determined by solving the two-dimensional Saint Venant equations with modifications to consider the effects of the temperature- and salinity-dependent density. The two-dimensional Saint Venant equations are, in fact, the vertically averaged Navier-Stokes equations. The WASH123D includes three 
approaches to the modeling of surface flow in a watershed system: the kinematic, diffusive, and dynamic wave models (Yeh et al. 2011). The fully dynamic wave model completely describes water flow but is sometimes difficult to solve under some conditions (e.g., when the slope of the ground surface is steep), regardless of what numerical approach is employed. On the other hand, the diffusion and/or kinematic models can handle a wide range of flow problems, but are inaccurate when the inertial terms play significant roles. Thus, three options are provided, the kinematic wave model, the diffusion wave model, and the dynamic wave model, to accurately compute the water flow over a wide range of conditions. The subsurface flow is described with the modified Richards equation. The modification incorporates the effects on density due to temperature and salinity. The governing equation is derived based on the continuity of fluid, continuity of solid mass, incompressibility of solids, and Darcy's law.

WASH123D was first developed by Yeh et al. (1998), and its design capability and flexibility have evolved. It has been applied in many projects world-wide including the Comprehensive Everglade Restoration Project (CERP) program. It has not only been chosen by the US Army Corps as the core computational code to model the Lower East Coast (LEC) Wetland Watershed, but has also been employed to construct a Regional Engineering Model for Ecosystem Restoration (REMER). A newly revamped WASH123D has been developed and applied to many of the river basins in Taiwan for flood and inundation forecasts for the purposes of disaster reduction (Shih and Yeh 2008; Shih et al. 2008; Liou et al. 2009; Yeh et al. 2011). In this study, the finite element approach is implemented for various spatial-temporal scale computations. The depth-averaged 2-D diffusive wave equations are solved to calculate the fluid flow in the watershed system (Yeh et al. 2006). The equations are solved with semi-Lagrangian and Galerkin finite element methods to determine coastal inundations. Various types of boundary conditions, determined based on physical reasoning, are essential to supplement the governing equations. Adequate data on initial conditions are obtained either from measurements or simulations of steady-state versions of the governing equations.

\section{MODELING CONFIGURATIONS}

This section includes the characteristics of the study sites, modeling configurations, computational procedures, and stability tests. The impact of the storm surge and overtopping with various return periods is calculated. The simulation of the breaking of a wave on a sloping beach is discussed in the text. We also explain why the solitary wave approach is adopted instead of using periodic wave modeling (Hsiao et al. 2008). The simulations are discussed in details below.

\subsection{Study Sites}

Coastal inundations may be influenced by several different factors, e.g., local topography, land cover, land subsidence, seawater run-ups, tides, and seawater intrusions, among others. Not all coastal areas will be subjected to inundations; the occurrence depends on local situations. This study is aimed at modeling extreme occurrences where the seawater intrudes into coastal areas or storm surge effects. The run-ups of breaking waves and the overtopping generated by drastic circumstances are assumed as boundary forcing for watershed modeling. The effects of precipitation are omitted in this study since it has been determined that it is oceanic effects that are the controlling factor. Seven townships, located along the eastern coast of Taiwan, were selected as our study sites (see Fig. 1). Land subsidence is not a crucial issue in most of the computational domains. Some jetties or moles have been partially constructed for protection. However, the inundations in low-lying coastal region tend to happen because of surface runoff running into the ocean often being obstructed by lifting tides. The intrusions of overtopping can cause local inundations, sometimes triggering major disasters in these littoral regions.

The study area also includes three low-lying coastal townships in Yilan County (Toucheng, Jiaoxi and Zhuangwei). The raised sea levels and spring tides often lead to a malfunctioning of the drainage system in these coastal townships. Toucheng Township has a lower elevation, for the most part below two meters, with some regions being even lower than sea level. Typhoon-induced storm surges and spring tides tend to obstruct flood run-off into the ocean. There is a high potential for inundation at the above mentioned sites. Sinchen Township is another site but with steeper terrain and sloping beaches where the greater danger is possible near the coast. Hualien County is another site of interest which has been identified to have higher terrain and unobvious land subsidence. This means less opportunity for extensive flooding throughout the entire area. However, overtopping and seawater intrusions are major disasters in terms of costs. Taitung County is also selected as another study site. In this area some jetties and protection barriers have been constructed and much steep terrain is identified. These engineering approaches do provide some effective protection to ease the problems of shoreline retreat and seawater intrusions. However, jetties along estuaries, especially near rivers have not been established yet. The final areas examined in this study where this is a problem are Taitung and Tamali Townships.

The computational domain was discretized using triangular elements to indicate land surface attributes. Each element was assigned a specific material to represent the heterogeneous characteristics. The surface elevation data for our study sites were extracted based on digitized aerial photographs taken at 40-meter intervals in 2002. Finer 

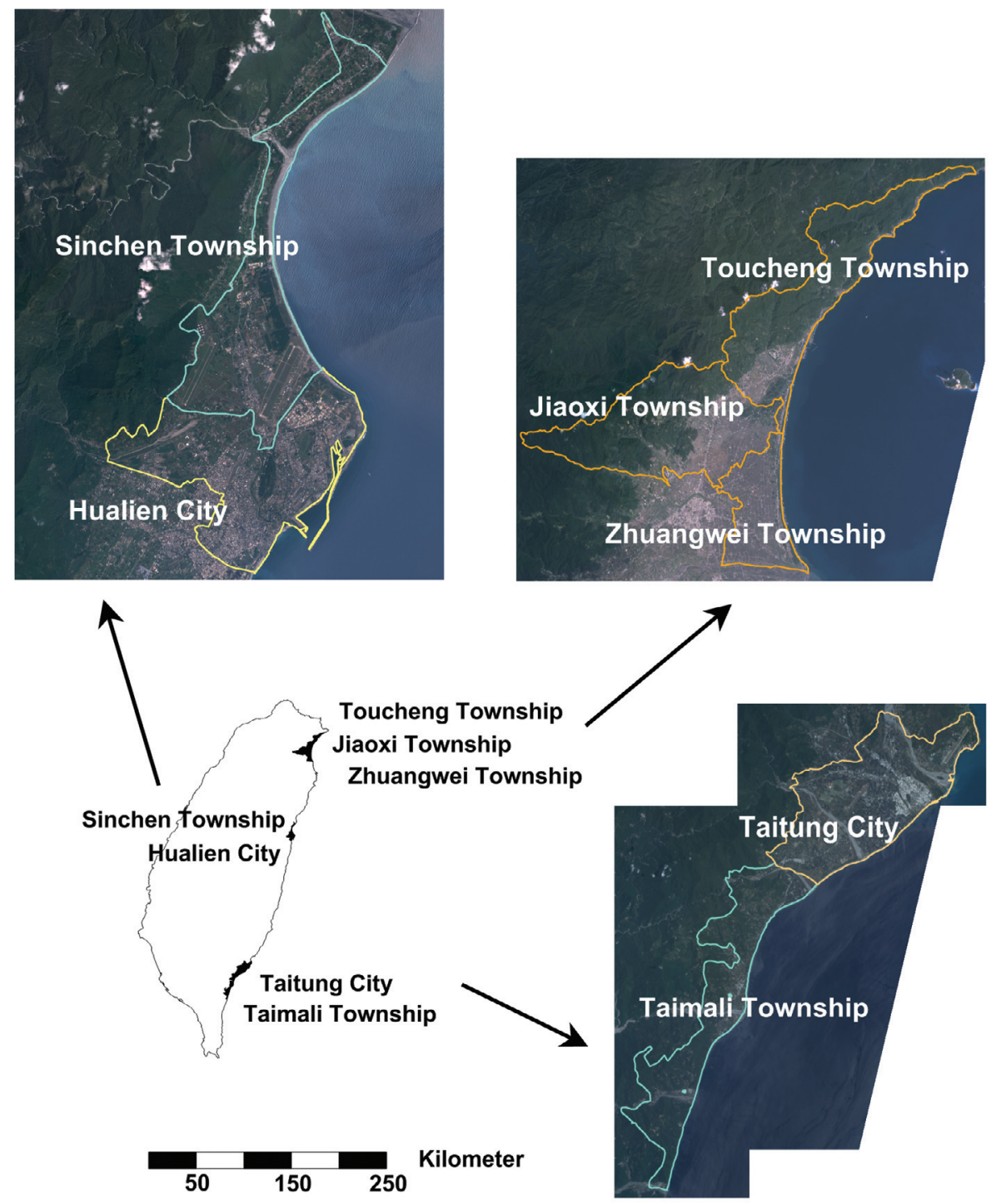

Fig. 1. Study sites.

grids were built near the coasts and coarser meshes were arranged inland in order to save computer time and memory storage. We built the main features artificially (e.g., rivers, roads, buildings, well-known landmarks) into the proposed computational domain. Then the digital elevation for each computational node was interpolated from the DTM data. Each type of mesh was assigned to represent a specific material based on the latest investigation of land cover carried out in 2006. The meshes were modified by comparison with satellite images from 2008. The Manning's coefficients for hydraulic routing were determined based on the attributes of each mesh. The values in each category were carefully computed based on specific sites (Shih and Yeh 2011). We used varied grid sizes to establish the main channels, drainage canals, riverbeds, and ponds. The appropriate bottom elevations and attributes were designated for each specific computational element after which the two-dimensional
WASH123D was used to conduct surface routing. The land use and the Manning's coefficients are categorized in Table 1. Based on the statistics, most land cover in the Yilan area can be categorized as belonging to the agriculture and forestry types. The majority of the coastal region is agricultural except for some windbreak forests cultivated along the littoral regions. Sinchen Township has cover mixed for the most part. Hualien City, on the other hand, is more urbanized than the other selected sites, with almost $30 \%$ being covered by buildings. The bulk of land cover in Taitung City is agriculture while the greater part of Taimali Township is related to forestry.

\subsection{Stability Testing and Modeling Configuration}

After discretization of the computational domain, stability tests were conducted for each study site. A model ex- 
amination was implemented using various types of boundary forcing. Astronomical tide data were first utilized to drive hydrological simulations to investigate mesh disposition. Figure 2 shows a plot of the astronomical tidal wave data applied as the downstream boundary condition in our watershed modeling.

The simulation results are plotted. Figure 3 a shows the extent of flooding within the Dezikou Stream drainage sys- tem. It does not make sense that the drainage system would malfunction when subjected only to astronomical tides. In addition, there is no mention in the literature or references revealing the circumstances for such inundations. We therefore conducted field investigations and reviewed government reports to find out the reasons. We found that a protective embankment along the Dezikou Stream drainage system had been constructed within the last few years.

Table 1. Land Use and Manning's roughness for the study sites.

\begin{tabular}{lcccccc}
\hline & Agricultural & Forestry & Traffic & Water & Buildings & Others \\
\hline Yilan area* & $32.01 \%$ & $51.44 \%$ & $3.54 \%$ & $3.08 \%$ & $5.04 \%$ & $4.89 \%$ \\
Sinchen Township & $27.93 \%$ & $13.06 \%$ & $6.04 \%$ & $4.28 \%$ & $9.92 \%$ & $38.77 \%$ \\
Hualien City & $14.35 \%$ & $9.08 \%$ & $14.30 \%$ & $5.77 \%$ & $28.37 \%$ & $28.13 \%$ \\
Taitung City & $50.78 \%$ & $6.53 \%$ & $7.00 \%$ & $7.75 \%$ & $8.43 \%$ & $19.51 \%$ \\
Taimali Township & $24.57 \%$ & $60.60 \%$ & $3.21 \%$ & $3.15 \%$ & $2.34 \%$ & $6.13 \%$ \\
Manning's N & 0.200 & 0.350 & 0.100 & 0.050 & 0.150 & 0.250 \\
\hline
\end{tabular}

* The Yilan area includes the Toucheng, Jiaoxi and Zhuangwei Townships in this study.

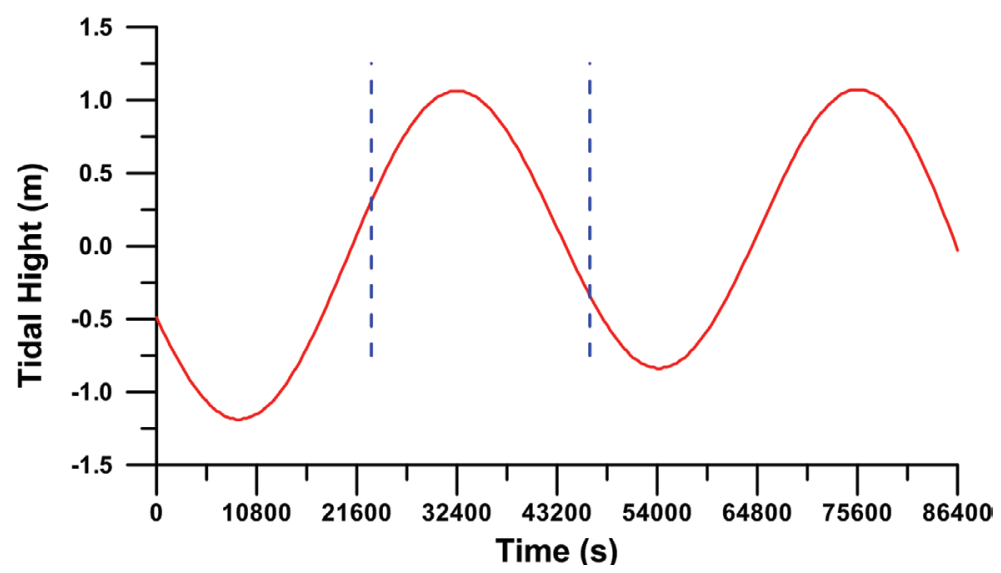

Fig. 2. Astronomical tidal wave data.

(a)

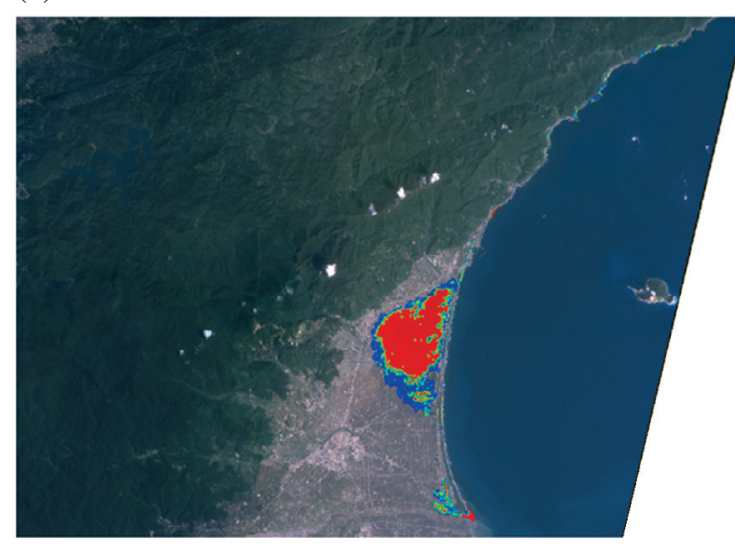

(b)

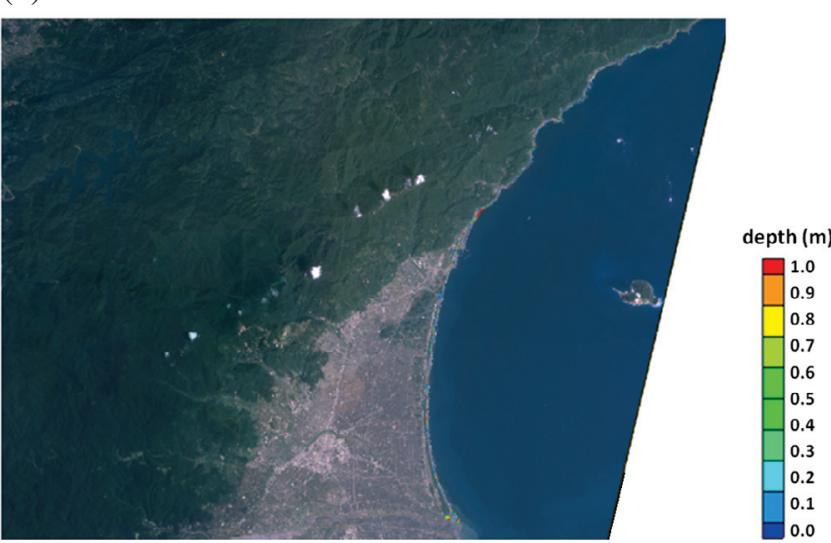

Fig. 3. Simulation using astronomical tidal waves as boundary forcing without dike (a) and with dike (b) discretization. 
The computational domain was then modified to include the embankments along the drainage system. The simulation results were much more reasonable compared to the results of previous mesh arrangements (see Fig. 3b). The influence of the astronomical tide affects only selected beaches along the coast. The simulation using astronomical tidal waves as boundary forcing reveals a much more reasonable response than dike discretization.

The results under the effects of astronomical tides for all remaining study sites, Sinchen Township, Hualien City,
Taitung City and Taimali Township, were reasonable with some minor flooding at only a few beaches. In Hualien City, very little water appeared on the estuary of the Meilun stream and Nan-Bai-Bin coastal areas, which is consistent with historical experience. There is higher potential for inundation in the estuary areas and low-lying areas near the coast. There were some slight side-effects identified in some coastal areas in the remaining regions; see Figs. 4 and 5.

We then continued testing, based on storm surges with various return periods. As stated above, the height of

(a)

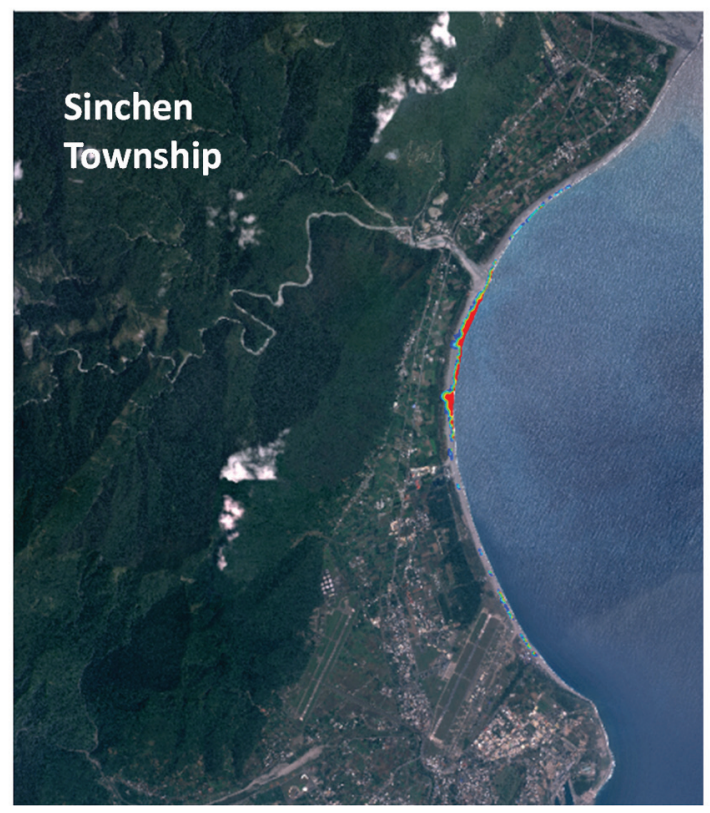

(b)

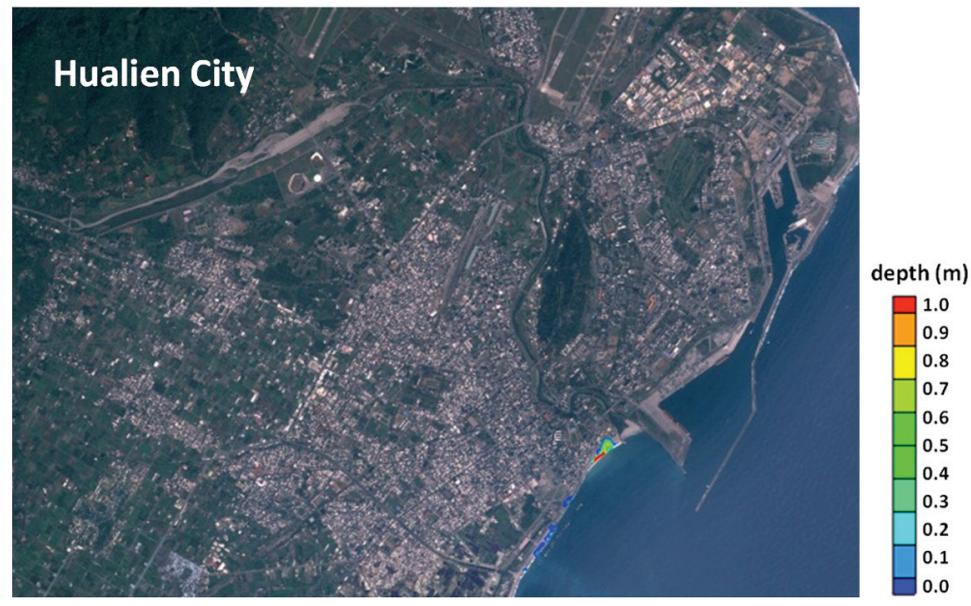

Fig. 4. Simulations using astronomical tidal movement as boundary forcing in Hualien County [Sinchen Township (a) and Hualien City (b)].

(b)

(a)
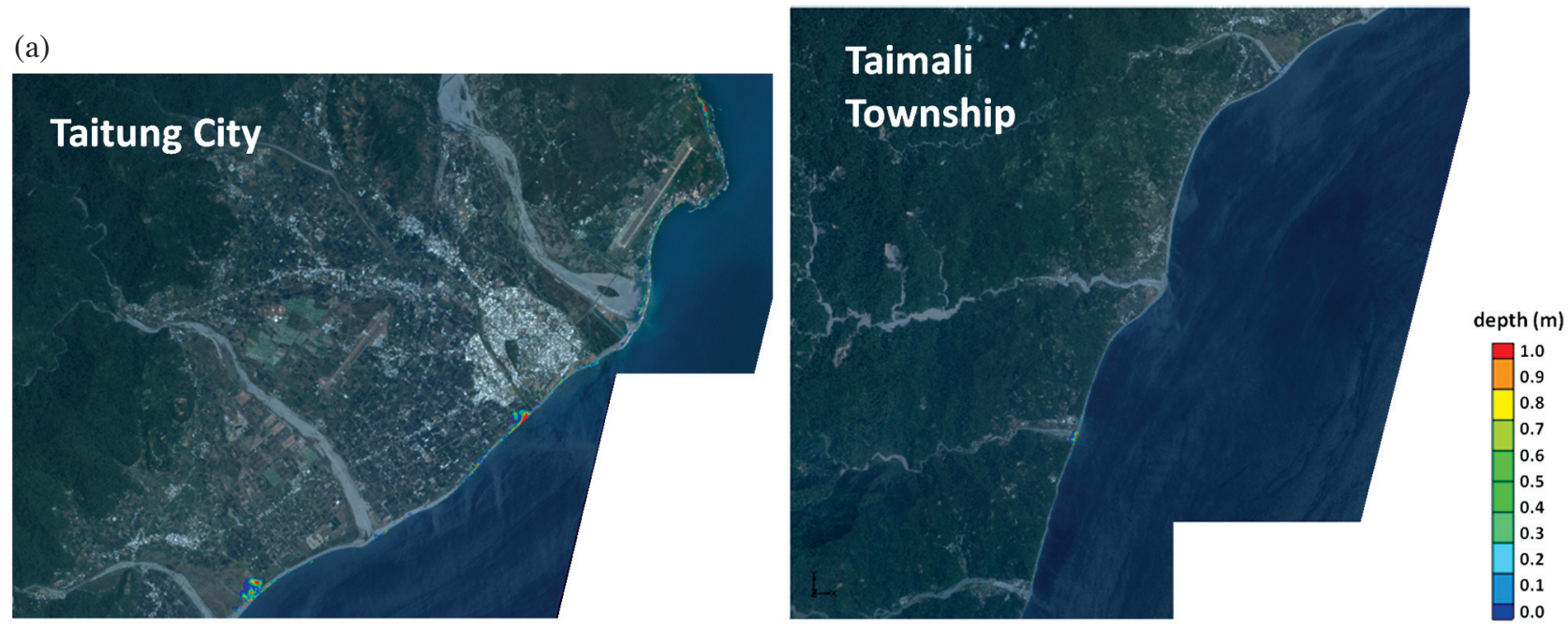

Fig. 5. Simulations using astronomical tidal movement as boundary forcing in Taitung County [Taitung City (a) and Taimali Township (b)]. 
waves generated by a storm surge is closely related to the local geography, land use, and the hydraulic characteristics of the watershed. Sea levels near the shore can be significantly elevated due to the effects of atmospheric pressure and strong winds during extreme events. Typhoon-induced storm surges occur frequently in Taiwan leading to coastal inundations. We applied goodness of fit tests to determine the appropriate statistical distribution of wave height which is different in each study. The results of statistical analysis of wave height including various return periods were then calculated, and are listed in Table 2. Simulation results of storm surges using boundary forcing with different return periods were examined, as shown in Figs. 6 to 10.

The simulation results showing storm surges in different return periods for Yilan County are plotted in Fig. 6. The simulations indicated that the windbreak forests in Zhuangwei and Toucheng Townships provided very good protection from the storm surges, with most of the water being stopped outside the windbreaks. Even for a storm surge with a 250-year return period there was only slight harm in this area, as shown in Fig. 6. There are two major drainage systems in the study domain, the Dezikou Stream and Yilan River. We found that the seawater could easily move through these channels under extreme storm surges. Seawater intrusion along the Dezikou stream occurred within the 20-year return period (see Fig. 6a). It was found that the Yilan river drainage system would be impacted by severe storm surges within a 50-year return period. Seawater intrusions due to extreme storm surges (return period of 250 years) occurred in both these drainage systems. No other large-scale inundation potential was detected.

Taking a closer look at the Dezikou Stream drainage system, it was revealed that in the extreme case, waves of more than 1 meter height could be intercepted by the windbreak forests, as shown in Fig. 7. We used satellite images to identify the extent of the windbreak forests, and then applied digital elevations to construct each computational node. The corresponding meshes were allocated to represent the windbreak forests. We found the elevated seawater was stopped outside the windbreaks, providing good protection along the coasts of Toucheng Township. Most seawater intrusions were found to occur along the Dezikou Stream drainage system. They would gradually move up along channels from downstream. Very little water, (depth of less than 10 centimeters) reached inland from the Dezikou Stream. Most intrusions due to flooding appeared in the downstream channels. No large-scale overflows were detected in this region. The well-constructed river dikes provided good protection to neighboring areas. Generally speaking, storm surges, even in the case with a 250-year return period, would rarely

Table 2. Statistics for wave height (m) with various return periods.

\begin{tabular}{llcccccccc}
\hline & & $\mathbf{5} \mathbf{~ y r}$ & $\mathbf{1 0} \mathbf{~ y r}$ & $\mathbf{2 0} \mathbf{~ y r}$ & $\mathbf{5 0} \mathbf{~ y r}$ & $\mathbf{1 0 0} \mathbf{~ y r}$ & $\mathbf{1 5 0} \mathbf{~ y r}$ & $\mathbf{2 0 0} \mathbf{~ y r}$ & $\mathbf{2 5 0} \mathbf{~ y r}$ \\
\hline Yilan area* & Gumbel & 1.65 & 1.80 & 1.94 & 2.12 & 2.26 & 2.34 & 2.40 & 2.44 \\
Sinchen Township & Weibull & 1.56 & 1.72 & 1.87 & 2.04 & 2.17 & 2.24 & 2.29 & 2.32 \\
Hualien City & Gumbel & 1.61 & 1.80 & 1.97 & 2.20 & 2.37 & 2.47 & 2.54 & 2.60 \\
Taitung City & Gumbel & 1.42 & 1.49 & 1.56 & 1.65 & 1.71 & 1.75 & 1.78 & 1.80 \\
Taimali Township & Gumbel & 1.47 & 1.54 & 1.62 & 1.72 & 2.17 & 2.24 & 2.29 & 2.32 \\
\hline
\end{tabular}

* The Yilan area includes the Toucheng, Jiaoxi and Zhuangwei Townships in this paper (Unit: meters).

(a)

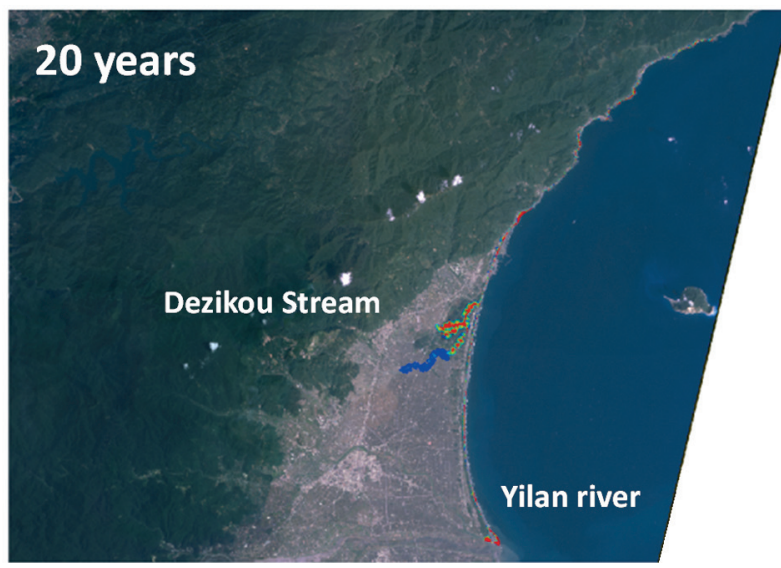

(b)

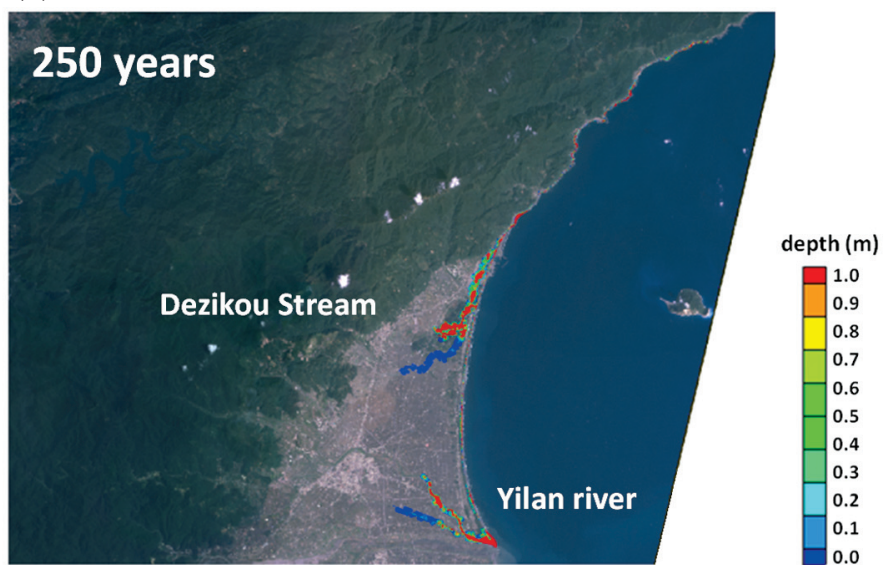

Fig. 6. Simulations using different return periods of storm surges as boundary forcing in Yilan County. 


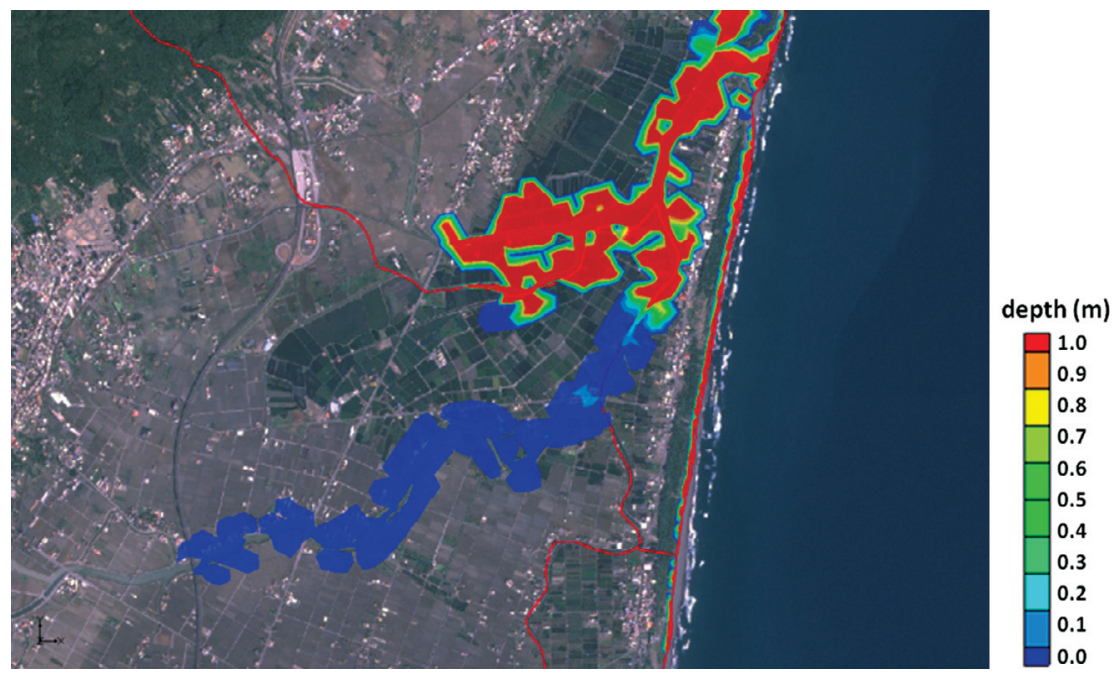

Fig. 7. Simulations using a 250 -year return period for storm surges as boundary forcing in the Dezikou Stream drainage system.

cause extensive damage in Toucheng Township. It is clear that the location of the river dikes and windbreak forests are appropriate.

Figure 8 offers a closer look at the simulation results for the Yilan River drainage system where even in extreme cases, run-ups of seawater intrusions could be intercepted by the windbreak forests. They provide good protection to the coastal area of Zhuangwei Township. Seawater might invade the upstream area of the Yilan River in the case of storm surges with a 50-year return period but we obtained running floods in the drainage system only around the estuary. Most intrusions of seawater flowed along the river drainage system. No large-scale overflows were detected in this region. The embankments provide good protection from storm surges. Even with a 250-year return period, such flooding would rarely cause extensive damage in Zhuangwei Township. The appropriate distributions of river dikes and windbreak forests have been set in our modeling.

Hualien City is a highly urbanized area. Engineering structures, such as building jetties to protect the population from storm surges, have been well established. The embankments constructed in the form of roads or artificial channels are also well designed to prevent overtopping. In our initial tests, we carried out simulations of extensive inundations using not only tidal waves but also storm surges. We found that the structured mesh arrangement failed to represent this domain. To more precisely model this domain, land cover and digital terrain had to be carefully examined. We used unsaturated grids to construct roads, artificial channels, and some of the main features. Protective structures including jetties, landmarks, and embankments were also carefully assigned. The appropriate attributes were allocated in the re-disposed domain. We obtained reasonable results when using the re-disposed meshes in the simulation (see Fig. 9). We found that the engineering structures provided the needed protection from storm surges of various degrees for return periods. Even in the extreme case with a 250-year of return period, the results showed that the embankments confined the inundations around coastlands that occurred to the regions near the Meilun Stream and Nan-Bin and BaiBin coastal areas. The Meilun Stream estuary is a low-lying area. There are some embankments in the form of roads in the Nan-Bin and Bai-Bin coastal areas which confined the floods resulting from storm surges along the coast. The majority of land cover is agricultural or low-lying estuary. No further influence was identified inland in residential areas.

Simulation results for the remaining computational domains, Sinchen Township, Taitung City and Taimali Township, are plotted in Fig. 10. The simulations show that there could be minor inundations along the coastline. Generally speaking, Sinchen Township, Taitung City and Taimali Township are regions with steep terrain with varying decreasing slopes from the interior to the coast. Consequently, seawater run-ups due to storm surges would rarely intrude inland with inundations caused by storm surges mostly obtained confined to the beaches. Most of the study sites have been identified as safe except for selected coastal areas.

\subsection{Scenario Discussion}

Watershed modeling integrated with wave overtopping and storm surges was conducted. Various scenarios were designed and are discussed in this section. We used the injected waves due to storm surges with different return periods as boundary forcing. The amount of overtopping for various return periods of 50,100, 150, 200 and 250 years was also calculated. Various simulated overtopping scenarios combined with storm surge data were simultaneously considered to evaluate the danger of surface inundation in the watershed modeling. We used the wind wave model to 


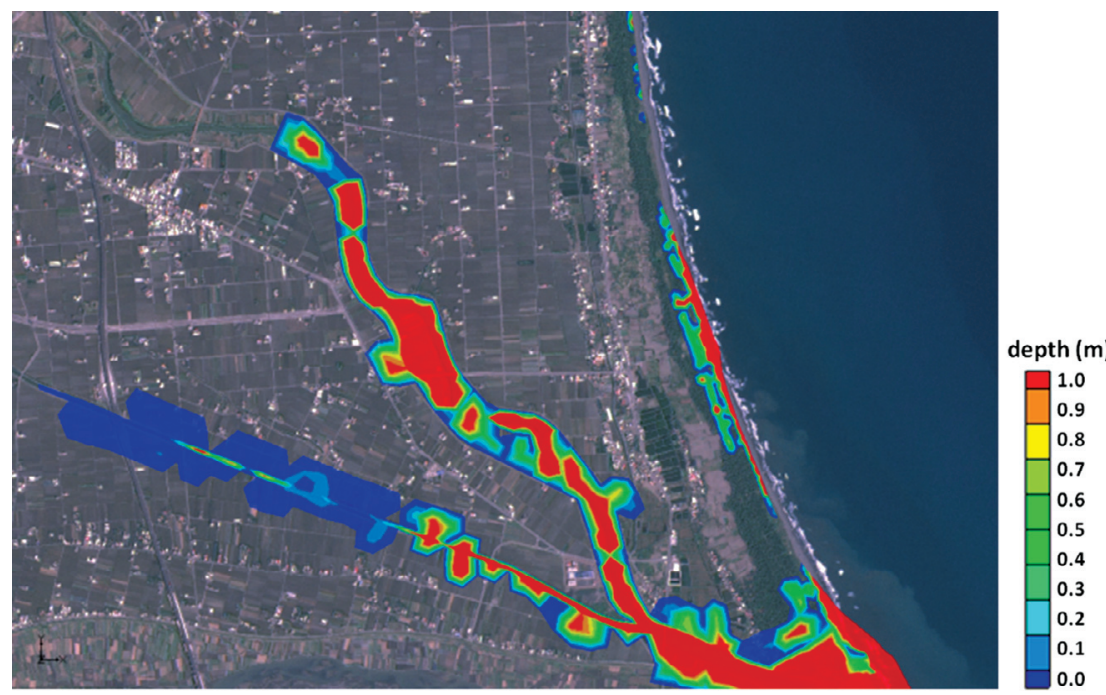

Fig. 8. Simulation using a 250-year return period of storm surges as boundary forcing in the Yilan River drainage system.

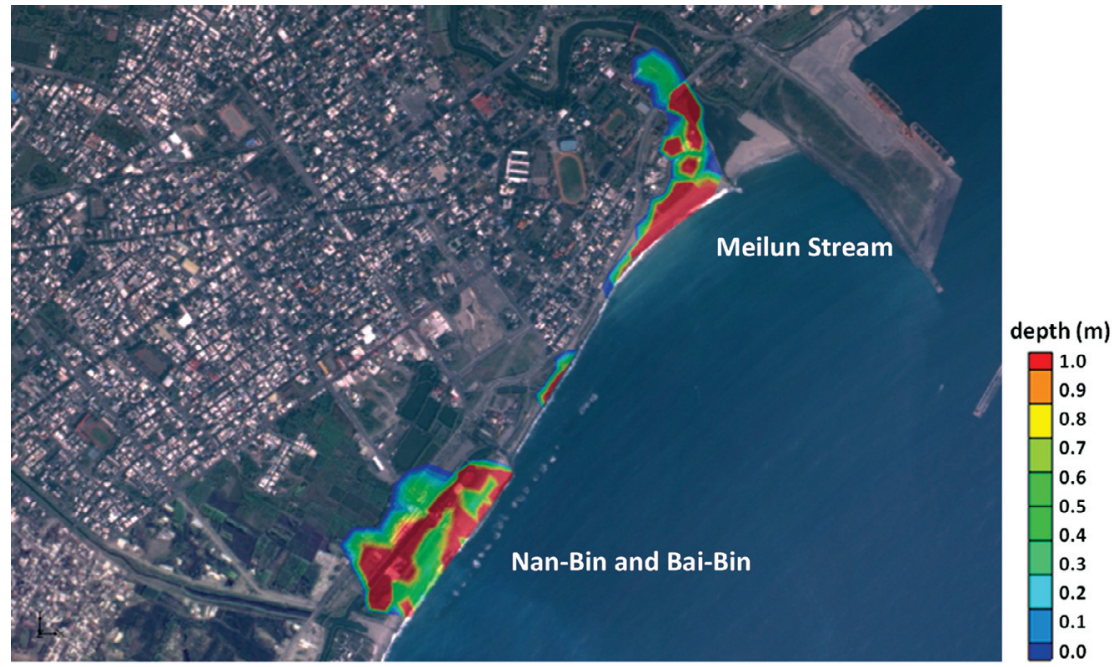

Fig. 9. Simulations using a 250-year return period of storm surges as boundary forcing in Hualien City.

(a)

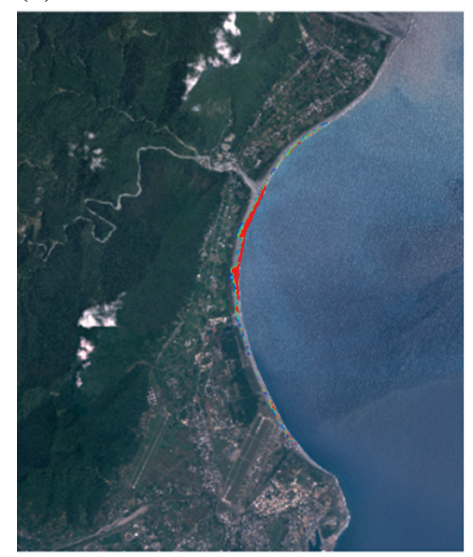

(b)

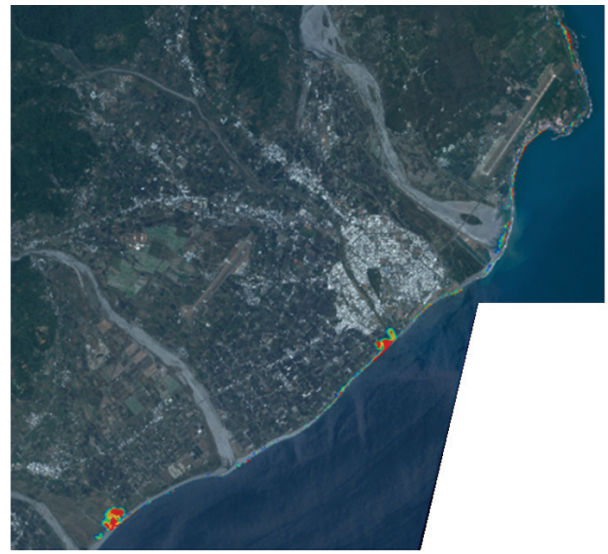

(c)

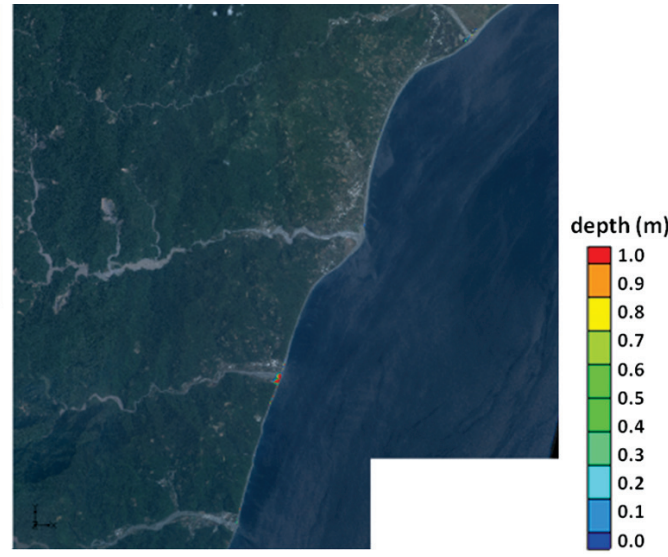

Fig. 10. Simulations using a 250-year return period with storm surges as boundary forcing in Sinchen Township (a), Taitung City (b) and Taimali Township (c). 
generate periodic waves which were continuously injected overland. However, overtopping as a consequence of wind wave modeling was not considered in the study sites. Although such an approach can more truly capture the characteristics of the waves, it was not suitable to apply the periodic wave modeling method to model overtopping in our computational domain of the east coast areas. Instead, we conducted the simulations using a solitary wave approach with vertically walled jetties. The quantities of overtopping in the study sites were eventually obtained. The overtopping and storm surge calculations were derived from one hour simulations of watershed modeling. More details of the simulations and a discussion are presented below.

Simulations integrating overtopping and storm surge scenarios with various return periods in Yilan County were conducted. Figure 11 shows the results of watershed simulations obtained using continuously injected overtopping and adopting a 250 -year return period as boundary forcing. It can be seen that only small amounts of water are identified in the channel in the first minute which gradually spreads from the estuary inland. However, the intrusion is still mainly confined inside the drainage system. After the passage of 10 minutes, more seawater has intruded and the water depth in the channels is getting higher. Now, inundation begins in the Dezikou drainage system and its neighboring regions, i.e., two sites on the Erlong River, along the banks of Xiapu and Dazuwei. The situation at the Yilan River is similar to the case for the Dezikou drainage system. Inundation spreads out to neighboring areas over the river bank becoming serious with increased simulation time. At the end of the model simulation, after the passage of 60 minutes, there was extensive seawater intrusion, overflowing from the Dezikou Stream and Yilan River drainage systems. In short, the model simulations suggest that the potential for inundation is high for areas along the Dezikou Stream and Yilan River, being low-lying regions in Yilan County. The bottom elevation in the drainage system is mostly below two meters with some channels being lower than sea level.
Surface inundations overflowing from the drainage system could thus occur. However, the flooding only spread out along the two sides of the channels. Extensive overflows throughout the entire city did not occur even in the case of extreme scenarios.

Figure 12 shows the results of watershed simulations incorporating overtopping and storm surges with 50-, $150-$, and 250-year return periods used as boundary forcing. Surface inundation was calculated for simultaneously simulated overtopping. According to the simulation results, inundation would typically take place along the coastline. Flooding could influence the south part of the estuary of the Sanzhan stream and outside of the airport. There was no influence inland. In short, inundation would be confined along the coast with no effects inland of Sinchen Township even under extreme situations.

Hualien City is the most urbanized site of all those investigated in this study. Surface inundations were examined assuming overtopping generated by the wind wave model with a return period of 250 years. Generally speaking, engineering structures constructed to prevent waves running up from the ocean were basically well-built and road embankments and artificial channels provided good local protection. Figure 13 shows the results with the injection of overtopping to the coastal areas of Hualien City after 10, 30 and 60 minutes of simulation time. Storm surges for the corresponding return periods were simultaneously applied to study the danger of surface inundation, including the extreme case with a return period of 250 years. Simulations show that inundations could occur in coastal areas around the estuary of the Meilun stream and Nan-Bin and Bai-Bin coastal areas. No extensive flooding was observed inland in Hualien City. Different forms of embankments (e.g., dikes, roads, schools, harbors) provide good protection for the city. Most inundations were identified to occur outside of the urban area.

However, the estuary of the Meilun stream is identified as having a high potential for flooding, being part of
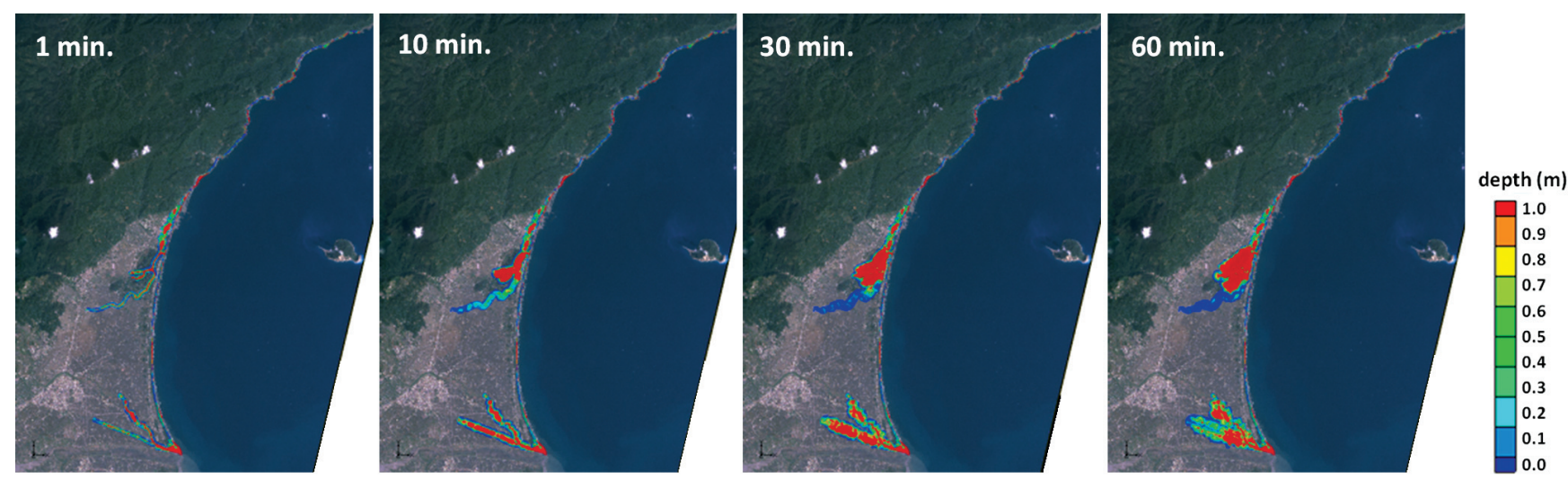

Fig. 11. Simulations integrating overtopping and storm surges for a 250-year return period in Yilan County. 

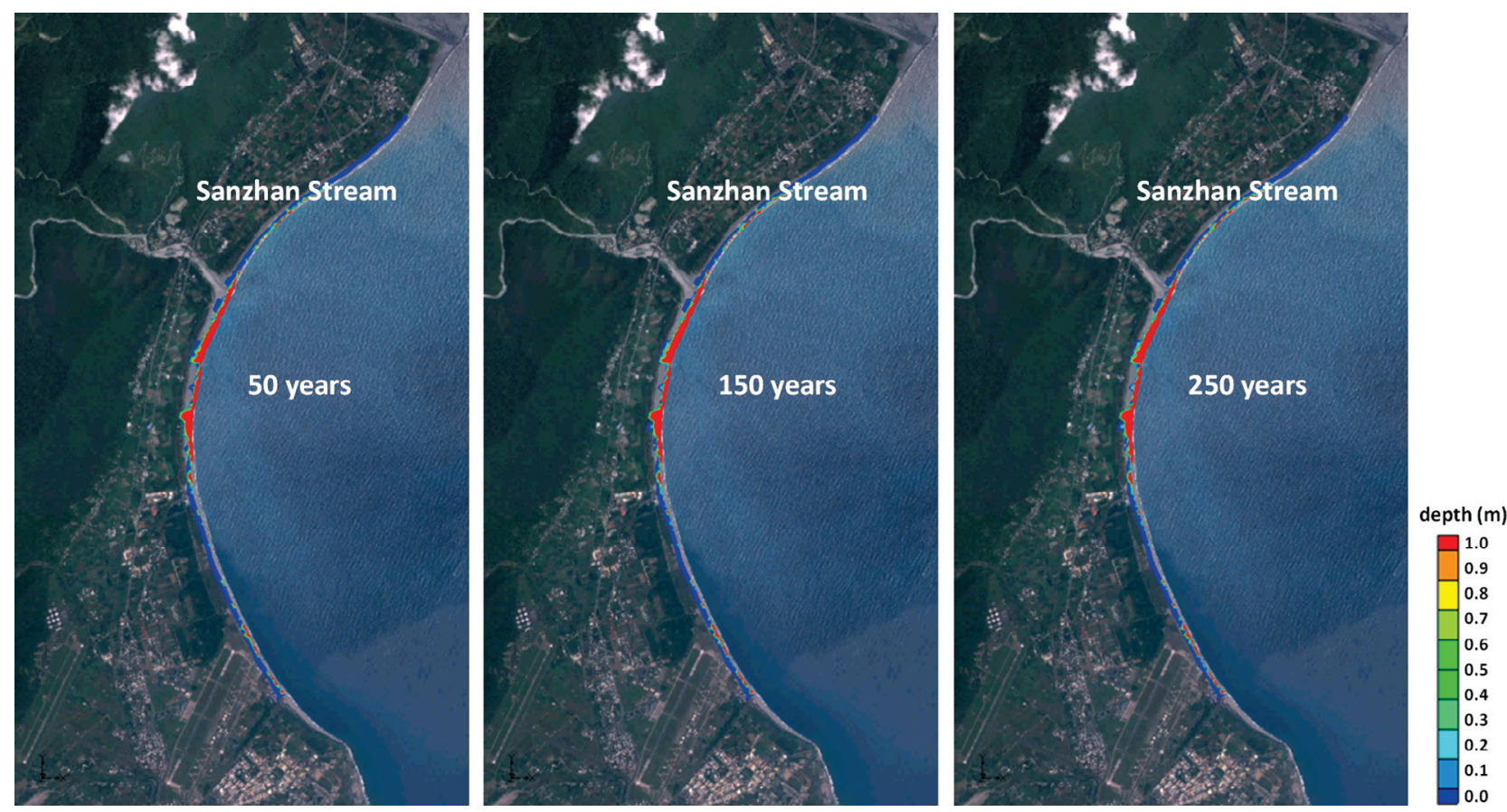

Fig. 12. Simulations integrating overtopping and storm surge using a return period of 250 years in Sinchen Township.
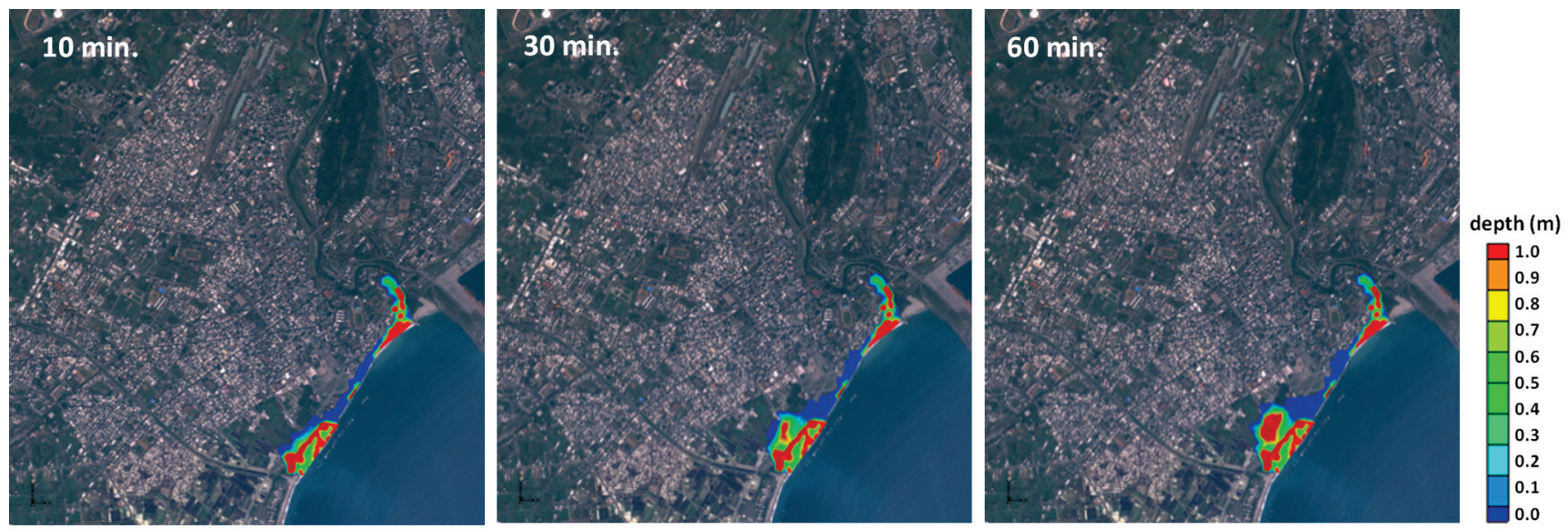

Fig. 13. Simulations integrating overtopping and storm surge with a return period of 250 years in Hualien City.

the low-lying area of the city where inundation can occur. In extreme cases, seawater could intrude along the Meilun stream. Increasing run-ups and overtopping would lead to inundation around the estuary with a gradual increase in water depth. The Nan-Bin and Bai-Bin coastal areas were also easily inundated by overtopping and storm surges. The simulations results show inundation in the Nan-Bin and BaiBin coastal areas after a time of 10 minutes of simulation. Thirty minutes later, the water depth becomes higher and also begins moving to the inland areas of Hualien City along the roads. The inundation keeps moving inland as time goes by. The regions covered by floods now extend outward. No further extensive inundation is obtained. Only some of the agriculture lands near the coast become inundated. Residential areas located inland of the city are rarely influenced. According to the model simulations, the flooding will be confined to the coasts between the estuary of the Meilun stream, and Nan-Bin and Bai-Bin coastal areas although seawater will intrude from the above regions in extreme scenarios. The agricultural lands near the coast and in the low-lying estuary deserve more attention. No significant damage was detected inland.

Figure 14 shows the simulation results for Taitung County. As stated above, storm surges with various return periods were used as boundary forcing. The overtopping was thus simultaneously integrated for study of flood routes. 


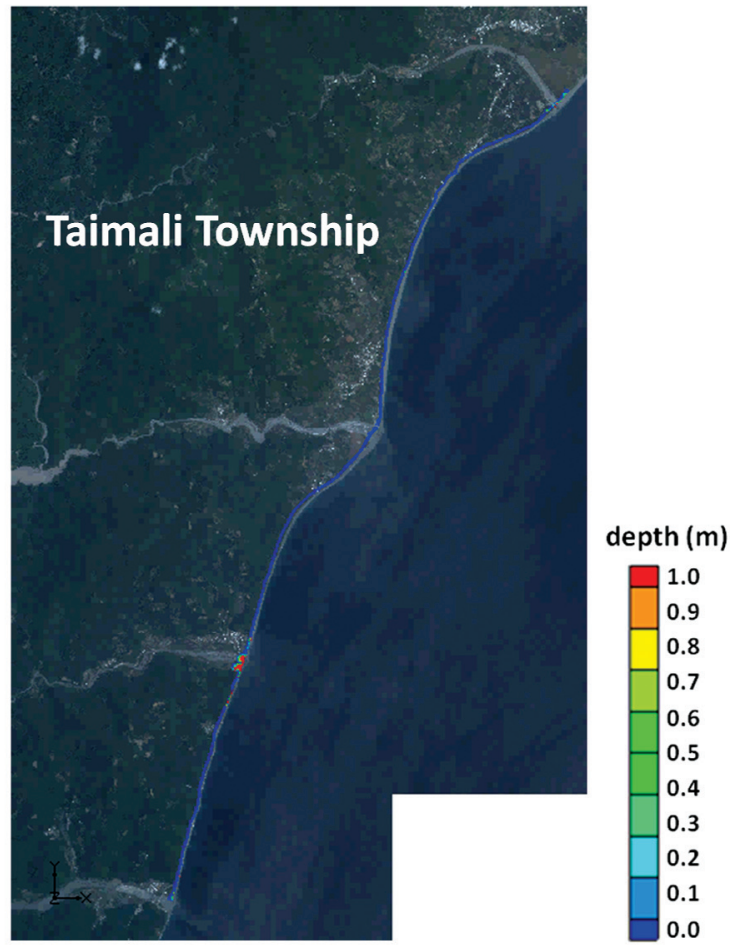

Fig. 14. Simulations with the integration of overtopping and storm surge for a return period of 250 years in Taitung County.

Simulations were similar to the calibrated cases. The extent of flooding along the coastlines is identified. The studied domains have steep terrain and decreasing altitudes so small amounts of wave run-ups and overtopping were found along the coastlines. We found some inundations, almost 1 meter, along the coastal areas of Taitung City. The satellite images show the locations of irrigation ponds and the local estuary. There were identified essentially as having a high potential for storing water. In general, the effects of extreme cases showed only a partial inundation of coastal areas. Even in extreme circumstances there was almost no harm to this region.

\section{SUMMARY}

The paper presents simulations of coastal flooding with a unified wind wave and a hydrological watershed model. Five sites in eastern Taiwan were studied. We used the Rankin-Vortex model and wind wave calculations to implement inputs for the simulations with overtopping and calculated storm surges as the boundary conditions in watershed modeling. The WASH123D was used to simultaneously integrate overtopping. The initial water levels for various return periods were used for model calibration. Data were accumulated from astronomical tide and storm surges. An examination of mesh discretization was conducted with inappropriate meshes at some computational sites corrected. Various scenarios integrating storm surges and overtopping were studied. We found the Dezikou Stream and Yilan River drainage systems in Yilan County have a high potential for inundation. Seawater could invade easily through the above channels in extreme circumstances. The extent of potential flooding in Hualien City included the coastal area between the Meilun Stream estuary and the coastal areas of Nan-Bin and Bai-Bin. We found the agricultural lands near the coast and in the low-lying estuary to be at higher risk. No significant danger of damage was detected for urban areas. Sinchen Township, Taitung City and Taimali Township are safer compared to the other study sites with the extent of inundation even in extreme cases only affecting the coast.

This study conducted simulations integrating wind wave and hydrological models for practical application in Taiwan. Simulations without precipitation were conducted, since overtopping has been determined to be the controlling factor. For further discussion of extreme circumstances, simulations of surface inundations due to combined precipitation and coastal wave effects are suggested.

Acknowledgements This research was supported in part by the Water Resources Agency, Taiwan, under Contract No. MOEAWRA0990228.

\section{REFERENCES}

Enright, D., R. Fedkiw, J. Ferziger, and I. Mitchell, 2002: A hybrid particle level set method for improved interface 
capturing. J.Comput.Phys., 183, 83-116. doi: 10.1006/ jcph.2002.7166. [Link]

Hsiao, S. C., T. W. Hsu, T. C. Lin, and Y. H. Chang, 2008: On the evolution and run-up of breaking solitary waves on a mild sloping beach. Coast. Eng., 55, 975-988, doi: 10.1016/j.coastaleng.2008.03.002. [Link]

Hsu, T. W., C. Y. Tsai, and J. W. Lai, 2009: RANS modeling of solitary wave propagation over a submerged rectangular breakwater. China Ocean Eng., 23, 473-488.

Hsu, T. W., J. M. Liau, J. G. Lin, J. Zheng, and S. H. Ou, 2011: Sequential assimilation in the wind wave model for simulations of typhoon events around Taiwan Island. Ocean Eng., 38, 456-467. doi: 10.1016/j.oceaneng.2010.11.023. [Link]

Liou, J. C., C. C. Chiang, and D. S. Shih, 2009: Establishing an operation system with unified regional circulation rainfall model and hydrological watershed model. AGU Fall Meeting, San Francisco, California, USA, 14-18 December 2009.

Shih, D. S. and G. T. Yeh, 2008: Numerical modeling of a mountainous watershed in Lanyang creek basin, Taiwan using WASH123d. The XVII International Conference on Computational Methods in Water Resources, San Francisco, California, USA, 6-10 July 2008.

Shih, D. S. and G. T. Yeh, 2011: Identified model parameterization, calibration, and validation of the physically distributed hydrological model WASH123D in Taiwan. J. Hydrol. Eng., 16, 126-136. doi: 10.1061/(AS
CE)HE.1943-5584.0000293. [Link]

Shih, D. S., J. M. Liau, and G. T. Yeh, 2008: Rainfall-runoff simulations with regional circulation rainfall model and hydrological watershed model. AGU Fall Meeting, San Francisco, California, USA, 15-19 December 2008.

Synolakis, C. E., 1987: The runup of solitary waves. $J$. Fluid Mech., 185, 523-545. doi: 10.1017/S00221120 8700329X. [Link]

Yeh, G. T., H. P. Cheng, J. R. Cheng, and H. C. Lin, 1998: A numerical model to simulate flow and contaminant and sediment transport in watershed systems (WASH12D). Technical Rep. CHL-98-15, Waterways Experiment Station, US Army Corps of Engineers, Vicksburg, Miss.

Yeh, G. T., G. B. Huang, F. Zhang, H. P. Cheng, and H. C. Lin, 2006: WASH123D: A numerical model of flow, thermal transport, and salinity, sediment, and water quality transport in WAterSHed systems of 1-D stream-river network, 2-D overland regime, and 3-D subsurface media. Technical Report, Submitted To EPA, Department of Civil and Environmental Engineering, University of Central Florida, Orlando, Florida, USA.

Yeh, G. T., D. S. Shih, and J. R. C. Cheng, 2011: An integrated media, integrated processes watershed model. Comput. Fluids, 45, 2-13. doi: 10.1016/j.compfluid.20 10.11.018. [Link] 